\section{Parenteral phosphate and amino acids supply effect on the growth of extremely preterm infants: accurate measurements and optimized statistical analysis are important}

\section{Dear Editor,}

We read with great interest the study by Moe et al. (1) that assessed the effect of different levels of parenteral phosphate and amino acids on the growth of preterm infants, using body weight as a surrogate for growth.

In preterm neonates, optimal parenteral intakes of energy, amino acids and minerals are necessary during the first days after birth to mitigate the sudden interruption of high intrauterine nutrient supply. This allows adequate protein synthesis to support body growth, including bone, using the intrauterine rate as a reference. However, body weight reflects body mass, which includes fat mass and a high proportion of water, especially in preterm neonates (2).

The variations in the supply of amino acid and phosphate would mainly have an impact on lean mass, including bone, which is better assessed clinically by crown to heel length than by weight. In fact, amino acids and phosphate are major determinants of the muscle and the bone matrix, and thus of skeletal growth. It is understandable that crown to heel length measurements were not considered sufficiently reliable in a retrospective analysis, because even prospectively accurate measurements in neonates are not easy to carry out.
Moe et al. (1) concluded that parenteral solutions with high amino acids and high phosphate content do not provide higher catch-up growth than solutions with high amino acids and low phosphate content. In addition to the graphical evidence, the authors base this conclusion on the statistical analysis of serial measurements of both serum phosphate and the weight standard score ( $Z$-score). A univariable analysis was performed based on the change in the weight $Z$-score from birth up to 29 days of age. The conclusions would have been stronger and more reliable if the analysis had taken into account three aspects: (i) the longitudinal character of the repeated measurements, that were available; (ii) the influence of factors that may also affect the outcome, such as being small for gestational age or receiving additional phosphate supply; and (iii) the interference from the lack of independence of the observations from twins and other multiples. Therefore, we consider that using mixed-effects regression models to carry out a multivariable analysis of the available longitudinal, repeated measurements would eventually demonstrate the lack of effectiveness of the solutions with high amino acids and high phosphate content more accurately than the performed analysis.

The results of this important study will encourage further research into which body compartments are more influenced by variations in amino acids and phosphate supply. Using noninvasive, accurate methods such as air displacement plethysmography and quantitative ultrasound to measure lean mass, segmental length and bone strength would provide more informative data. Furthermore, friendlier and potent statistical tools that can be used to analyse longitudinal data have recently become available, and these allow more powerful statistical hypotheses tests and more accurate estimates of effect size (3).

\section{References}

1. Moe K, Beck-Nielsen S, Lando A, Greisen G, Zachariassen G. Administering different levels of parenteral phosphate and amino acids did not influence growth in extremely preterm infants. Acta Paediatr 2015; 104: 894-9.

2. Pereira-da-Silva L, Virella D. Is intrauterine growth appropriate to monitor postnatal growth of preterm neonates? BMC Pediatr 2014; 14: 143.

3. Gibbons R, Hedeker D, DuToit S. Advances in analysis of longitudinal data. Annu Rev Clin Psychol 2010; 6: 79-107.

\section{DOI:10.1111/apa.13153}

Daniel Virella (danielvirella@chlc.min-saude. pt ${ }^{1,2}$, Luis Pereira-da-Silva ${ }^{1,2}$, Ana Luisa Papoila ${ }^{1,3}$ 1.Research Unit, Centro Hospitalar de Lisboa Central, Lisbon, Portugal

2.NICU, Hospital de Dona Estefânia, Centro Hospitalar de Lisboa Central, Lisbon, Portugal

3.Biostatistics and Informatics Department, NOVA Medical School, Universidade Nova de Lisboa, Lisbon, Portugal

\section{Correspondence}

D Virella, Centro de Investigação do Centro Hospitalar de Lisboa Central, Hospital de Dona Estefânia, Rua Jacinta Marto, 1169-045 Lisboa, Portugal.

Tel: +351936361793 |

Fax: +351213596443 |

Email: danielvirella@chlc.min-saude.pt 